\title{
Reatividade em ambiente de contenção móvel: uma nova metodologia para avaliar o temperamento bovino
}

\author{
[Animal reactivity in a mobile cage: a new methodology to access bovine temperament]

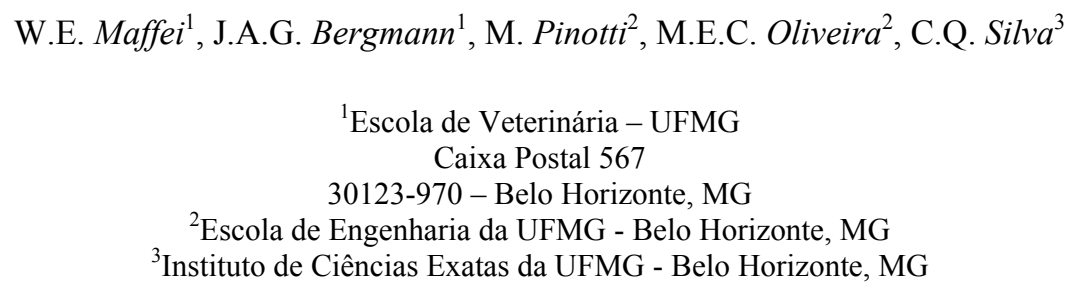

\section{RESUMO}

Descreve-se uma nova metodologia para medir o temperamento dos bovinos por meio da reatividade, obtida por um dispositivo eletrônico que quantifica o número e a intensidade dos movimentos do animal em ambiente de contenção móvel, numa escala de 1 a 9.999 pontos. A reatividade foi comparada a características determinadas por outros dois métodos tradicionais para quantificar o temperamento bovino: a velocidade de fuga e o escore de temperamento. Foram utilizados 610 bovinos da raça Nelore, com média de idade de 467 dias e provenientes de dois rebanhos. As observações da reatividade não se ajustaram às distribuições normal, Poisson e binomial negativa. A padronização e as transformações logarítmicas neperianas e na base 10 não foram eficientes para adequar os dados à distribuição normal. Os animais avaliados para a característica apresentaram grande variabilidade fenotípica, com reatividade média de $534 \pm 447$ pontos na escala original, mediana 442 pontos, moda 372 pontos, variação de 1 a 2.218 pontos e coeficiente de variação de $84 \%$. A correlação de classificação de resíduos encontrada entre reatividade e escore de temperamento variou de 0,75 a $0,85(\mathrm{P}<0,001)$ e foi superior em valores absolutos às correlações entre reatividade e velocidade de fuga e entre velocidade de fuga e escore de temperamento. A nova metodologia é eficiente para quantificar o temperamento de animais da raça Nelore e possui vantagens em relação às características velocidade de fuga e escore de temperamento. A obtenção da reatividade animal é rápida, prática, objetiva e segura para quem a faz, além de apresentar grande variabilidade fenotípica.

Palavras-chave: bovino, reatividade animal, temperamento

\begin{abstract}
This paper describes a new methodology to access bovine temperament using it's reactivity eletronically measured as the intensity and count of movements in a mobile cage, using a scale from 1 to 9,999 points. The new trait, reactivity, was compared to two other traditional methods, flying speed and temperament scores. Six hundred and ten contemporary Nellores, with average age of 467 days and from two herds were used. Reactivity data did not fit Normal nor Poisson nor Negative Binomial Distibutions. Standartized reactivity data, neperian and base 10 log reactivity transformations were not successful in adjusting data to normal distribution. Reactivity presented large phenotipic variation, with average in the original scale of 534 \pm 447 points, median of 442, mode 372, range from 1 to 2,218 points and variation
\end{abstract}

Recebido em 2 de janeiro de 2006

Aceito em 18 de setembro de 2006

E-mail: wemaffei@uol.com.br 


\section{Maffei et al.}

coefficient of $84 \%$. Residual rank correlation between reactivity and temperament score vary from .75 to $.85(P<.001)$, and were larger than the ones between reactivity and flight speed and between flight speed and temperament score. The new methodology is efficient in measuring temperament of Nellore cattle and it has advantages in relation to the traits flight speed and temperament score. The measure of reactivity is easy, fast to get and safe to record. In addition, this trait presents large variability.

Keywords: bovine, animal reactivity, temperament

\section{INTRODUÇ̃̃O}

Cerca de $80 \%$ do rebanho bovino nacional é de origem Zebu, predominantemente Nelore. Apesar de sua importância para a pecuária de corte, a raça Nelore apresenta algumas limitações, como o temperamento mais agressivo que o de raças taurinas.

Definido como a expressão do comportamento de medo em resposta às ações realizadas pelo homem durante as atividades de manejo diário com os animais (Fordyce e Burrow, 1992), o temperamento influencia as características produtivas, reprodutivas e organolépticas da carne. Animais de mau temperamento provocam acidentes com outros animais e com vaqueiros, aumentam os custos de manutenção das benfeitorias e têm pior qualidade do couro (Gomes, 1997; Cardoso, 2001).

Como os estudos realizados para estimar o coeficiente de herdabilidade encontram valores de 0,26 a 0,67 , diversos autores propõem a inclusão da característica temperamento nos programas de melhoramento genético (Sato, 1981; Csiro, 1988; Burrow, 1991; Fordyce e Burrow, 1992), o que possibilitaria diminuir a agressividade dos animais e, conseqüentemente, melhorar a qualidade em toda cadeia produtiva da carne.

Apesar de sua importância, a quantificação do temperamento bovino não é tarefa fácil. $\mathrm{O}$ escore de temperamento, método utilizado para medir a característica de forma subjetiva, avalia o grau de perturbação do animal quando manejado no tronco, no curral ou em pastagem, baseando-se em ações comportamentais como o vigor e a freqüência da movimentação, da respiração, dos movimentos de cauda, da ocorrência de coices e das expressões faciais (Fordyce et al.,1982; Mourão et al., 1999). Dois métodos objetivos também foram propostos e utilizados para quantificar o temperamento dos bovinos, a velocidade de fuga (Burrow et al., 1988) e a distância de fuga (Fordyce et al., 1982). Menores tempos para o primeiro e maiores distâncias para o segundo são indicativos de animais com temperamento mais agressivo (Burrow et al., 1988; Fordyce et al., 1982).

Esses métodos, que foram desenvolvidos e têm sido aplicados em raças de origem européia ou em mestiços europeu-zebu, apresentam limitações, pela dificuldade de aplicação, principalmente quando se trata de animais zebus.

O presente trabalho apresenta uma nova metodologia para quantificar de forma objetiva o temperamento bovino, a reatividade em ambiente de conteção móvel. A característica reatividade, obtida por meio de um dispositivo eletrônico que quantifica a intensidade e o número de movimentos do animal em ambiente de contenção móvel, foi descrita e comparada com as características tradicionais velocidade de fuga e escore de temperamento.

\section{MATERIAL E MÉTODOS}

Foram utilizados 610 animais da raça Nelore, sendo 367 (182 fêmeas e 185 machos) com média de idade de 445 dias, provenientes da Fazenda Santa Marta, localizada em Naviraí, MS, e 243 (135 fêmeas e 108 machos) com média de idade de 490 dias, provenients da Fazenda Perdizes, localizada em Campo Grande, MS. 
Os animais da Fazenda Santa Marta foram criados juntos até a desmama, realizada, em média, aos sete meses de idade. Após a desmama, machos e fêmeas foram separados em grupos de contemporâneos e recriados em pastejo rotacionado. Os animais da Fazenda Perdizes obedeceram ao mesmo manejo, porém, os machos foram recriados em pastejo rotacionado e as fêmeas em pastejo semiintensivo.

Três características foram utilizadas como indicadoras do temperamento bovino, assim descritas: A - Velocidade de fuga - medida objetiva do tempo, em décimos de segundo (ds), que o animal leva para percorrer a distância de 1,7 metros, após sair de um espaço confinado, como o brete de uma balança ou o tronco, em direção a um espaço aberto. A medida foi realizada com o auxílio de duas células fotoelétricas localizadas na saída do brete, a uma altura de 1,2 metros do solo. O tempo gasto foi armazenado em uma unidade receptora de tempo. B - Escore de temperamento - medida subjetiva, adaptada da metodologia proposta por Fordyce et al. (1982) e Mourão et al. (1999). Nessa adaptação, os animais foram fechados no brete sem contenção, e a freqüência e o vigor dos movimentos foram avaliados numa escala que variou de 1 a 5, descrita como se segue: 1. temperamento muito dóci 1- animal estático, em geral encostado na parte da frente ou de trás do brete, com movimentos de cauda ocasionais e relaxados; 2. temperamento dócil - animal calmo, mas não permanecendo na mesma posição por mais do que alguns segundos e com movimentos de cauda ocasionais; 3. temperamento levemente agressivo - animal com deslocamentos freqüentes dentro do brete, movimentos vigorosos e abruptos, movimentos de cauda freqüentes e vigorosos e respiração audível; 4. temperamento agressivo - animal com deslocamentos quase contínuos dentro da balança, movimentos vigorosos e abruptos, respiração audível, mas sem presença de mugido; 5. temperamento muito agressivo - animal com deslocamentos contínuos dentro da balança, apresentando saltos e forçando a grade do brete com a cabeça ou posterior, movimentos de cauda contínuos e vigorosos com ocorrência de mugidos e bufar pesado.
Reatividade animal em ambiente de contenção móvel - medida da reatividade do animal (em pontos), por meio de um dispositivo eletrônico acoplado ao brete (Maffei, 2004). O dispositivo é dotado de um mecanismo que quantifica a freqüência e a intensidade da movimentação do animal em 20 segundos. Esse tempo, estabelecido após a realização de alguns prétestes, teve início assim que o animal entrou no brete e o portão foi fechado. O dispositivo eletrônico, desenvolvido na Universidade Federal de Minas Gerais (Maffei, 2004), teve depósito de patente no Instituto Nacional de Propriedade Industrial, como Patente Modelo de Utilidade - № DEINPI/MG 001088.

Nas duas fazendas, a rotina de pesagem dos animais não foi alterada para a obtenção da reatividade. A única instrução dada aos vaqueiros foi a de que eles não poderiam tocar no brete no momento da quantificação da reatividade, tomada durante a pesagem dos animais.

Assim como para a reatividade, os escores de temperamento também foram obtidos observando o animal por 20 segundos no momento da pesagem. A característica velocidade de fuga foi obtida na saída do brete, logo após o término da quantificação da reatividade, do escore de temperamento e da pesagem do animal.

Inicialmente, as observações das características reatividade e velocidade de fuga foram submetidas à análise descritiva para a obtenção das medidas de tendência central e de dispersão (média, mediana, moda, valores máximo e mínimo, amplitude, variância e desvio padrão). Além disso, as observações da reatividade animal foram dispostas em histogramas, para visualização de possível distribuição. As observações do escore de temperamento, por serem categóricos ordinais, foram apresentadas em um diagrama setorial com a freqüência por escore.

A etapa seguinte consistiu da avaliação da distribuição de probabilidade das observações da reatividade animal. Para isso, foram verificadas as adequações das observações à distribuição 
normal, à distribuição de Poisson e à distribuição binomial negativa.

A adequação à distribuição normal foi avaliada por meio da análise do histograma e do teste de normalidade de Ryan-Joiner, incluído no pacote estatístico Minitab (2000). A adequação às distribuições de Poisson e binomial negativa foi avaliada por meio do software STATA, utilizado para ajustar as observações aos respectivos modelos e do software SPLUS para realizar o teste qui-quadrado da qualidade do ajuste aos modelos. A hipótese nula adotada, de que a característica reatividade seguia a distribuição proposta, foi testada no nível de $5 \%$ de probabilidade.

Após as análises de adequação das observações às distribuições Poisson e binomial negativa, e constatado que as observações não se adequaram a nenhuma delas, optou-se por adotar a distribuição normal e por fazer algumas transformações de variáveis para a obtenção de propriedades da distribuição normal. Assim, as observações foram submetidas à padronização (desvio da média dividido pelo desvio-padrão) e a transformações logarítmicas, que, segundo Sampaio (2002), são as mais adequadas para variáveis biológicas que possuem desvio-padrão com variação proporcional à média, como foi o caso da reatividade animal. As transformações logarítmicas são dadas por $\log ^{(r)}$, em que (r) é a reatividade animal. Nas transformações utilizaram-se logaritmo neperiano e logaritmo na base 10

A última etapa consistiu da verificação da associação da reatividade com a velocidade de fuga e o escore de temperamento do animal. Foi inicialmente estimada a correlação de classificação dentro de fazenda por meio do pacote estatístico SAS (User's..., 1998). Para isto, as observações na escala contínua da reatividade e da velocidade de fuga foram também categorizadas numa escala com variação de 1 (menor reatividade ou maior tempo) a 5 (maior reatividade ou menor tempo). A escala categórica foi determinada para cada fazenda, a partir da amplitude da característica dividida em cinco classes, de acordo com a expressão: $\frac{\text { Amplitude da característica }}{5}=$ Intervalo das categorias

A categorização resultante foi a seguinte:

Fazenda Santa Marta

Categoria 1: Reatividade $\leq 317$ pontos ou velocidade de fuga $\geq 76 \mathrm{ds}$;

Categoria 2: Reatividade $\geq 318$ e $\leq 635$ pontos ou velocidade de fuga $\geq 57$ e $\leq 75 \mathrm{ds}$;

Categoria 3: Reatividade $\geq 636$ e $\leq 953$ pontos ou velocidade de fuga $\geq 38$ e $\leq 56 \mathrm{ds}$;

Categoria 4: Reatividade $\geq 954$ e $\leq 1.271$ pontos ou velocidade de fuga $\geq 19$ e $\leq 37 \mathrm{ds}$; e

Categoria 5: Reatividade $\geq 1.272$ pontos ou velocidade de fuga $\leq 18 \mathrm{ds}$.

\section{Fazenda Perdizes}

Categoria 1: Reatividade $\leq 503$ pontos ou velocidade de fuga $\geq 136 \mathrm{ds}$;

Categoria 2: Reatividade $\geq 504$ e $\leq 943$ pontos ou velocidade de fuga $\geq 102$ e $\leq 135 \mathrm{ds}$;

Categoria 3: Reatividade $\geq 944$ e $\leq 1.383$ pontos ou velocidade de fuga $\geq 68$ e $\leq 101 \mathrm{ds}$;

Categoria 4: Reatividade $\geq 1.384$ e $\leq 1.823$ pontos ou velocidade de fuga $\geq 34$ e $\leq 67 \mathrm{ds}$; e

Categoria 5: Reatividade $\geq 1.824$ pontos ou velocidade de fuga $\leq 33 \mathrm{ds}$.

Foram obtidas correlações de classificação entre as características escore de temperamento, reatividade e velocidade de fuga do animal, as duas últimas nas escalas original e categórica.

Outra abordagem sobre a associação entre escore de temperamento e as características reatividade e velocidade de fuga foi obtida a partir da modelagem das observações dessas características em função do escore de temperamento, considerado como variável independente, por meio do procedimento PROC GLM do pacote estatístico SAS (User's..., 1998).

A visualização gráfica da variação observada para cada característica foi obtida por meio de Box Plot e diagrama de dispersão para cada fazenda, considerando para ambos, a associação entre os pontos de reatividade e o escore de temperamento atribuído a cada animal. 


\section{RESULTADOS E DISCUSSÃO}

$\mathrm{O}$ teste de reatividade em ambiente de contenção móvel foi realizado na fazenda Santa Marta nos dias 13 e 14 de março de 2003. Cento e dois animais foram avaliados no dia 13, das $18 \mathrm{~h} 15 \mathrm{~min}$ às $19 \mathrm{~h} 7 \mathrm{~min}$, com média de tempo de duração para obtenção da reatividade de cada animal de 39 segundos. $\mathrm{O}$ restante dos animais foi avaliado no dia 14 de março, 81 deles avaliados das $8 \mathrm{~h} 15 \mathrm{~min}$ às $9 \mathrm{~h} 8 \mathrm{~min}$, com média de tempo de duração para cada animal de 40 segundos, 75 das $9 \mathrm{~h} 56 \mathrm{~min}$ às $10 \mathrm{~h} 45 \mathrm{~min}$, com média de tempo de duração para cada animal de 39 segundos e, finalmente, 106 das $14 \mathrm{~h} 12 \mathrm{~min}$ às $15 \mathrm{~h} 16 \mathrm{~min}$, com média de tempo de duração para cada animal de 37 segundos. $\mathrm{Na}$ fazenda Perdizes, o teste foi realizado nos dias 17 e 18 de março de 2003, sendo 135 animais avaliados no dia 17 , das $14 \mathrm{~h} 35 \mathrm{~min}$ às $16 \mathrm{~h} 20 \mathrm{~min}$, com média de tempo de duração para cada animal de 47 segundos, e o restante, 83 , no dia 18 de março, das $7 \mathrm{~h} 55 \mathrm{~min}$ às $8 \mathrm{~h} 40 \mathrm{~min}$, com média de tempo de duração para cada animal de 52 segundos. O reduzido tempo necessário para obtenção da característica e a ausência de interferência dessa prática com a obtenção de peso dos animais, prática corriqueira nos rebanhos de bovinos de corte, ilustra a aplicabilidade da obtenção da reatividade bovina.

Os animais avaliados apresentaram grande variabilidade para as características reatividade e velocidade de fuga, indicadoras do temperamento, com elevados coeficientes de variação. As diferenças observadas para as três medidas de tendência central indicam desvio para a esquerda na distribuição das duas características (Tab. 1).

Para a característica escore de temperamento, os percentuais de animais incluídos nas categorias $1,2,3,4$ e 5 foram, respectivamente, 17, 16, 27, 19 e $21 \%$. A maioria dos animais avaliados, $67 \%$, teve escore igual ou superior a 3 , isto é, de temperamento levemente agressivo a muito agressivo.
Tabela 1. Estatística descritiva das características reatividade animal e velocidade de fuga de animais da raça Nelore

\begin{tabular}{lcc} 
Estatística & $\begin{array}{c}\text { Reatividade } \\
\text { (pontos) }\end{array}$ & $\begin{array}{c}\text { Velocidade de } \\
\text { fuga (décimos de } \\
\text { segundo) }\end{array}$ \\
\hline Média & 534 & 15,9 \\
Mediana & 442 & 14 \\
Moda & 372 & 14 \\
Variância & $199.692,7$ & 245,4 \\
Desvio-padrão & 446,9 & 15,7 \\
Coeficiente de & 84 & 99 \\
variação, \% & & \\
Mínimo & 1 & 1 \\
Máximo & 2.219 & 164 \\
\hline
\end{tabular}

Observou-se que nenhum dos perfis das observações de reatividade dispostas em histograma individuais para cada fazenda, ou em conjunto, aproximou-se da distribuição normal (Fig. 1), o que foi comprovado pela rejeição da hipótese nula $(\mathrm{P}<0,05)$. Esta situação já havia sido constatada pela avaliação das informções da Tab. 1, pois, segundo Doria Filho (1999), para que uma amostra de dados se adeque à distribuição normal, sua média, mediana e moda devem coincidir ou estarem muito próximas.

Como as observações da reatividade não se adequaram à distribuição normal, e por caracterizarem como do tipo numérico discreto, testou-se sua adequação às distribuições de Poisson e binomial negativa, com rejeição $(\mathrm{P}<0,05)$ da hipótese nula para ambas. De forma semelhante, a padronização das informações e as trasformações testadas (logarítmica neperiana e a transformação logarítmica na base 10) não adequaram as observações da reatividade animal à distribuição normal $(P<0,05)$. Assim, todas as análises subseqüentes para comparar a característica escore de temperamento com as características velocidade de fuga e reatividade foram feitas na escala original. Deve-se salientar que a totalidade dos trabalhos encontrados que relatam características indicadoras do temperamento animal apresenta análises na escala original (Maffei, 2004). 


\section{Maffei et al.}
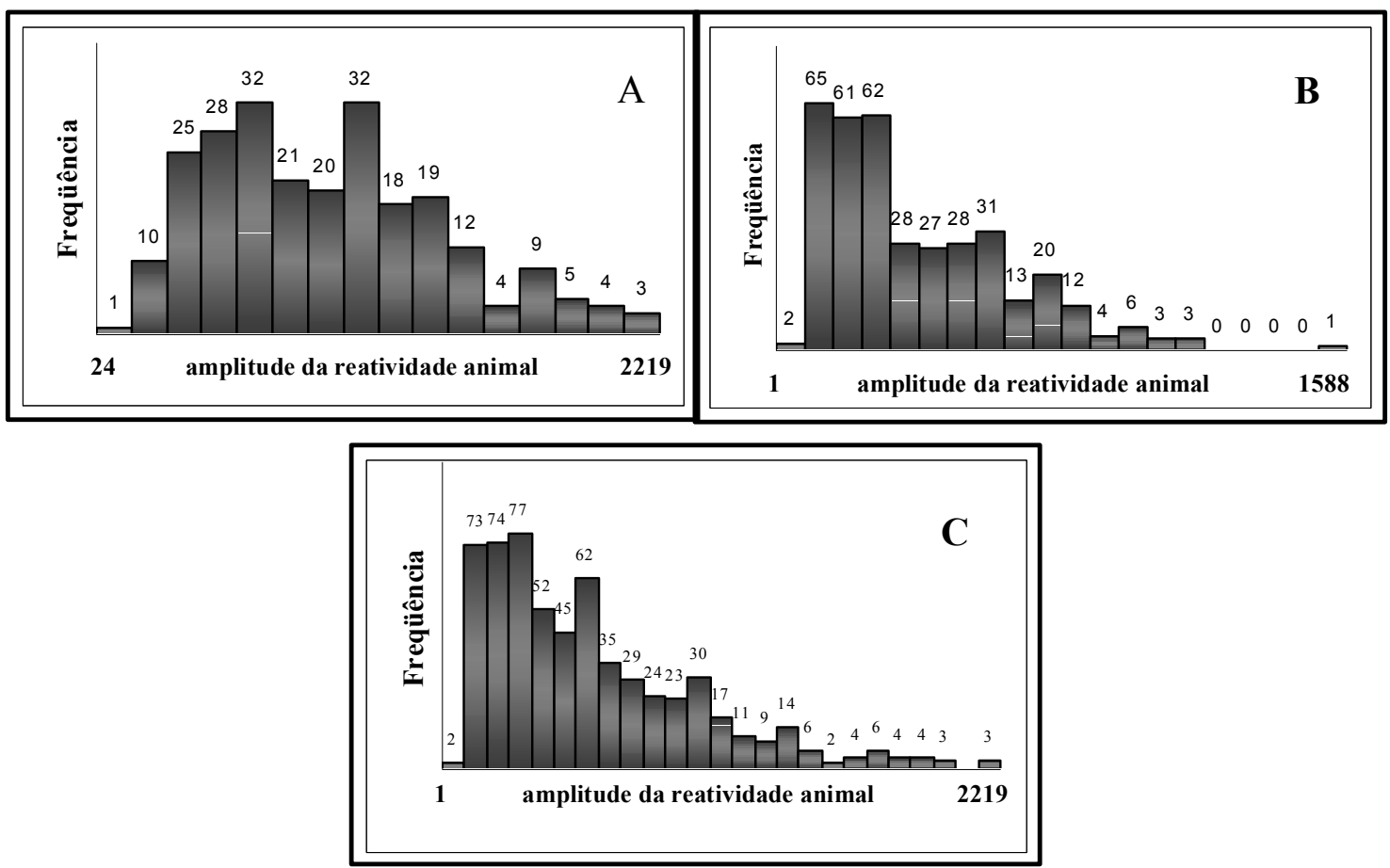

Figura 1. Histograma da reatividade de animais da raça Nelore nas Fazendas Santa Marta (A), Perdizes (B) e em conjunto (C).

As correlações de classificação entre as características reatividade, observada na escala contínua, e escore de temperamento foram elevadas, 0,85 na Fazenda Santa Marta e 0,82 na Perdizes, indicando que os animais classificados com maiores pontuações para reatividade também apresentaram temperamento mais agressivo e vice-versa. O mesmo ocorreu quando reatividade foi categorizada, porém com valores menores, 0,75 na Fazenda Santa Marta e 0,78 na Perdizes (Tab. 2). Estes resultados evidenciam elevada associação entre as duas características. Já a associação entre escore de temperamento e velocidade de fuga não ocorreu de forma tão evidente. Para as observações originais ela foi de $-0,41$ na Fazenda Santa Marta e -0,22 na Perdizes, e para as observações da velocidade de fuga classificadas em categorias, observaram-se 0,26 e 0,04 , respectivamente. É relevante mencionar que o sinal negativo da correlação entre os dados na escala contínua da velocidade de fuga e o escore de temperamento indica que quanto menor o tempo observado para a velocidade de fuga, mais agressivo seria o animal. Em relação à característica reatividade, foi evidente a menor magnitude das associações entre as observações da velocidade de fuga e do escore de temperamento, indicando que os animais classificados como de menores tempos na velocidade de fuga não necessariamente tiveram os maiores escores de temperamento.

A associação entre velocidade de fuga e reatividade dos animais também foi baixa. Para as Fazendas Santa Marta e Perdizes, os valores das correlações foram, respectivamente, -0,34 e 0,18 , quando as duas características estavam na escala contínua; 0,17 e -0,02, quando as duas características estavam categorizadas; 0,21 e 0,01 , quando reatividade estava na escala contínua e velocidade de fuga categorizada; e 0,29 e -0,14, quando reatividade estava categorizada e velocidade de fuga na escala contínua. Sendo assim, os animais com menores tempos para a velocidade de fuga não necessariamente tiveram as maiores pontuações para reatividade. 
Tabela 2. Correlações entre as características reatividade animal observada na escala contínua e em cinco categorias, de 1 (menor reatividade) a 5 (maior reatividade), velocidade de fuga, observada na escala contínua e em cinco categorias, de 1 (menor velocidade) a 5 (maior velocidade), e escore de temperamento de animais da raça Nelore das Fazendas Santa Marta (acima da diagonal) e Perdizes (abaixo da diagonal)

\begin{tabular}{|c|c|c|c|c|c|}
\hline \multirow{2}{*}{$\begin{array}{l}\text { Características e } \\
\text { escala }\end{array}$} & \multicolumn{2}{|c|}{ Reatividade } & \multicolumn{2}{|c|}{ Velocidade de fuga } & \multirow[t]{2}{*}{$\begin{array}{c}\text { Escore de } \\
\text { temperamento }\end{array}$} \\
\hline & Contínua & Categorizada & Contínua & Categorizada & \\
\hline \multicolumn{6}{|l|}{ Reatividade } \\
\hline Contínua & - & $0,89 * *$ & $-0,34 * *$ & $0,21 * *$ & $0,85^{* *}$ \\
\hline Categorizada & $0,96^{* *}$ & - & $-0,29 * *$ & $0,17 * *$ & $0,75 * *$ \\
\hline \multicolumn{6}{|l|}{ Velocidade de fuga } \\
\hline Contínua & $-0,18 * *$ & $-0,14^{*}$ & - & $-0,72 * *$ & $-0,41^{* *}$ \\
\hline Categorizada & $-0,01$ & $-0,02$ & $-0,45^{* *}$ & - & $0,27 * *$ \\
\hline $\begin{array}{l}\text { Escore de } \\
\text { temperamento }\end{array}$ & $0,82 * *$ & $0,78 * *$ & $-0,22 * *$ & 0,04 & - \\
\hline
\end{tabular}

Os resultados da correlação de classificação para a Fazenda Santa Marta foram semelhantes aos da Fazenda Perdizes (Tab. 2), indicando que em ambas os animais com maiores pontuações para reatividade também apresentaram maiores escores de temperamento. Já os animais com menores tempos para a velocidade de fuga não necessariamente tiveram os maiores escores de temperamento $\mathrm{e}$ as maiores pontuações para reatividade.

Os resultados confirmam que a reatividade pode ser utilizada como um indicativo do temperamento de animais da raça Nelore (Tab. 3), uma vez que as médias ajustadas foram estatisticamente diferentes para cada escore de temperamento. Por outro lado, a velocidade de fuga não seria um bom indicativo do temperamento animal, pois as médias ajustadas da característica para os diferentes escores de temperamento não diferiram estatisticamente, sobrepondo-se e inclusive com uma inversão.

Sabe-se que, por ser subjetivo, o escore de temperamento é mais sujeito a erros de avaliação do que as características medidas de forma objetiva. $\mathrm{Na}$ avaliação dos animais pelo escore de temperamento é fácil separar os extremos, ou seja, diferenciar um animal muito dócil, possivelmente com escore 1, de um muito agressivo, possivelmente com escore 5. Porém, a diferenciação entre os animais com escores intermediários é mais difícil. Esta dificuldade pode ser visualizada nas Fig. 2 e 3, pois em ambas as fazendas os extremos de temperamento, escores $1 \mathrm{e}$ 5 , foram excludentes para pontos de reatividade, enquanto os intermediários não o foram.

Aliada aos problemas de se avaliar o temperamento bovino por meio de um método subjetivo, a Fig. 3 ilustra outra vantagem da característica reatividade sobre o escore de temperamento, a sua maior variabilidade fenotípica. Assim, o escore de temperamento 1, por exemplo, englobou animais com reatividade de 1 a 218 e de 24 a 959 pontos, respectivamente nas Fazendas Santa Marta e Perdizes.

Tabela 3. Médias ajustadas pelo método dos quadrados mínimos e erros-padrão da reatividade e velocidade de fuga de animais da raça Nelore, incluindo-se o escore de temperamento como variável independente na escala de 1 (muito dócil) a 5 (muito agressivo)

\begin{tabular}{ccc}
\hline $\begin{array}{c}\text { Escore de } \\
\text { temperamento }\end{array}$ & Reatividade animal (pontos) & Velocidade de fuga (ds) \\
\cline { 2 - 3 } & Média \pm erro-padrão & Média \pm erro-padrão \\
\hline 1 & $206,86 \pm 23,97 \mathrm{a}$ & $19,76 \pm 1,53 \mathrm{a}$ \\
3 & $362,86 \pm 24,29 \mathrm{~b}$ & $18,54 \pm 1,54 \mathrm{ab}$ \\
4 & $517,50 \pm 18,78 \mathrm{c}$ & $15,82 \pm 1,23 \mathrm{bc}$ \\
5 & $733,66 \pm 22,31 \mathrm{~d}$ & $14,28 \pm 1,43 \mathrm{c}$ \\
\hline
\end{tabular}

Médias seguidas de letras distintas, na coluna diferem entre si $(\mathrm{P} \geq 0,05)$. 

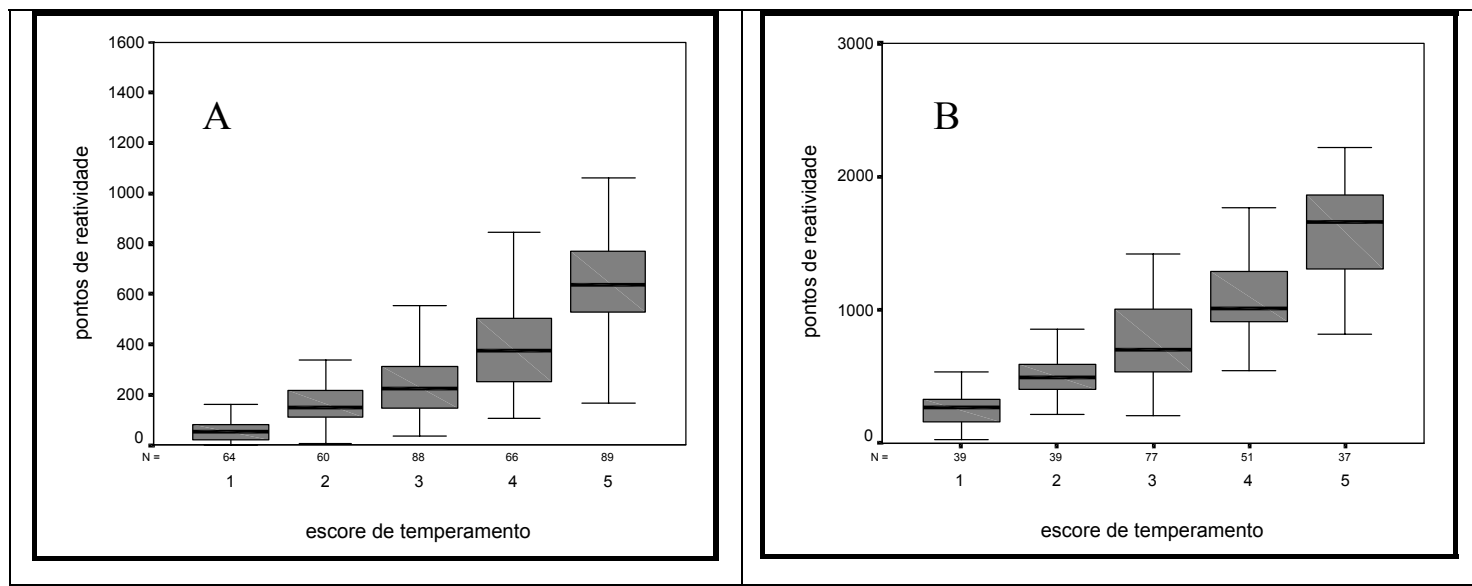

Figura 2. Box Plot da reatividade animal em função do escore de temperamento de animais da raça Nelore, nas Fazendas Santa Marta (A) e Perdizes (B).

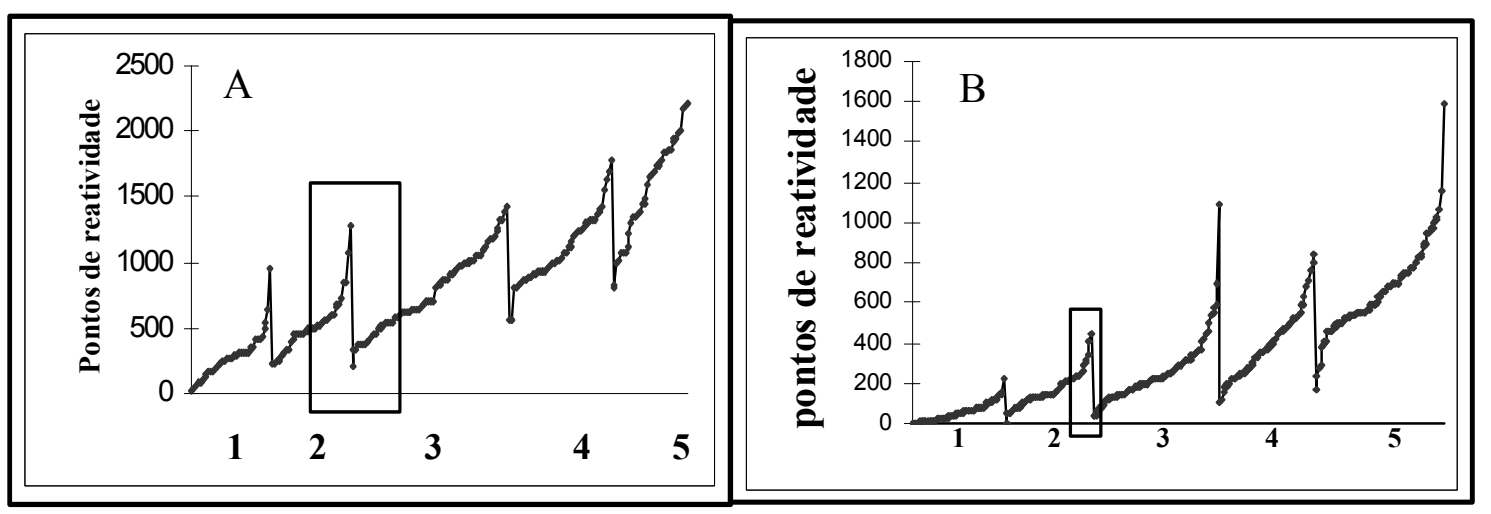

Figura 3. Diagrama de dispersão da reatividade animal em função do escore de temperamento de animais da raça Nelore, nas Fazendas Santa Marta (A) e Perdizes (B).

\section{CONCLUSÕES}

A metodologia teste de reatividade animal em ambiente de contenção móvel tem aplicação rápida, prática e segura para quem a utiliza, e é eficiente para quantificar, de forma objetiva, a reatividade de animais da raça Nelore. O teste tem grande aplicabilidade, já que seu dispositivo eletrônico pode ser acoplado a qualquer tipo de brete móvel, como os de balanças individuais ou coletivas, eletrônicas ou mecânicas. A facilidade para realização do teste, que pode ser realizado no momento da pesagem dos animais, possibilita conduzi-lo sem alterar a rotina das práticas de manejo dos rebanhos bovinos de corte. A característica reatividade apresenta grande variabilidade fenotípica e está associada ao escore de temperamento, sendo mais adequada para indicá-lo que a velocidade de fuga.

\section{REFERÊNCIAS BIBLIOGRÁFICAS}

BURROW, H.M. Effect of intensive handling of zebu crossebred weaner calves on temperament In: CONFERENCE OF THE AUSTRALIAN ASSOCIATION ANIMAL BREEDING GENETICS, 9., 1991, Victória. Proceedings..., Victória, 1991. p.208-211.

BURROW, H.M.; SEIFERT, G.W.; CORBET, N.J. A new technique for measuring temperament in cattle. Proc. Aust. Soc. Prod., v.17, p.154-157, 1988.

CARDOSO, E.E. Sistemas integrados de produção de peles e couros no Brasil. 2001. Disponível em: 
$<$ http://www.cnpgc.embrapa.br/publicação/doc/doc 127/12sistemas.html> Acessado em: 04/dez/2003.

CSIRO. Genetic and environment methods of improving the temperament of Bos indicus and crossbred cattle. In: AUSTRALIAN MEAT RESEARCH COMITTEE, 1988, Queensland. Final report..., Queensland, 1988.

DORIA FILHO, U. Introdução à bioestatística: para simples mortais. 3.ed. São Paulo: Negócio Editora, 1999. 158p.

FORDYCE, G.; BURROW, H. Temperament of Bos indicus bulls and its influence on reproductive efficiency in the tropics. In: WORKSHOP BULL FERTILITY, 1., 1992, Rockhampton. Proceedings..., Rockhampton, 1992. p.35-37.

FORDYCE, G.; GODDARD, M.E.; SEIFERT, G.W. The measurement of temperament in cattle and the effect of experience and genotype. Anim. Prod., v.14, p.329-332, 1982.

GOMES, A. Como melhorar a qualidade do couro. $1997 . \quad$ Disponível em: $<$ http://www.snagricultura.org.br/artigos/artitecbovinos.html> Acessado em: 04/dez/2003.
MAFFEI, W.E. Reatividade animal em ambiente de contenção móvel-um método alternativo para quantificar o temperamento bovino. 2004. 32f. Dissertação (Mestrado em Zootecnia) - Escola de Veterinária, Universidade Federal de Minas Gerais, Belo Horizonte.

MINITAB Realease 13.0. Copyright 2000, Minitab INC.

MOURÃO, G.B.; BERGMANN, J.A.; MADALENA, F.E. et al. Diferenças genéticas e estimação de coeficientes de herdabilidade para características morfológicas em fêmeas zebus e F1 Holandês-Zebu. Rev. Bras. Zootec., v.28, p.44-54, 1999.

SAMPAIO, I.B.M. Estatística aplicada à experimentação animal. 2.ed. Belo Horizonte: FEPMVZ, 2002. 265p.

SATO, S. Factors associated with temperament of beef cattle. Jpn. J. Zootec. Sci., v.52, p.595-605, 1981.

USER'S guide: statistics. Version 8.0, Cary, NC: SAS Institute, 1998. 